\title{
The genetics of juvenile idiopathic arthritis: Searching for new susceptibility loci
}

\author{
MARIA I. ZERVOU ${ }^{1 *}$, DESPOINA G. DIMOPOULOU $^{2 *}$, ELIAS ELIOPOULOS $^{3}$, MARIA TRACHANA $^{4}$, \\ POLYXENI PRATSIDOU-GKERTSI ${ }^{4}$, ATHENA ANDREOU ${ }^{3}$, PRODROMOS SIDIROPOULOS ${ }^{5}$, \\ DEMETRIOS A. SPANDIDOS ${ }^{6}$, ALEXANDROS GARYFALLOS ${ }^{2}$ and GEORGE N. GOULIELMOS ${ }^{1}$
}

\begin{abstract}
${ }^{1}$ Section of Molecular Pathology and Human Genetics, Department of Internal Medicine, School of Medicine, University of Crete, Heraklion $71003 ;{ }^{2}$ th Department of Internal Medicine, Hippocratio Hospital, Aristotle University of Thessaloniki, Thessaloniki 54124; ${ }^{3}$ Laboratory of Genetics, Department of Biotechnology, Agricultural University of Athens, Athens 11855; ${ }^{4}$ st Department of Pediatrics, Aristotle University of Thessaloniki, Thessaloniki 54124; ${ }^{5}$ Department of Rheumatology, Clinical Immunology and Allergy, University Hospital of Heraklion, Heraklion 71500;

${ }^{6}$ Laboratory of Clinical Virology, School of Medicine, University of Crete, Heraklion 54124, Greece
\end{abstract}

Received September 1,2017; Accepted October 2, 2017

DOI: $10.3892 / \mathrm{mmr} .2017 .7733$

\begin{abstract}
Juvenile idiopathic arthritis (JIA) is an autoimmune disease that is characterized by persistent chronic arthritis and affected by genetic and environmental factors. Different genetic variations have been reported as risk factors for JIA. However, given that many results could not be replicated in individuals of different ancestral origin, it was assumed that heterogeneous genetic factors are involved in this disease. In the present study, we analyzed three single nucleotide polymorphisms (SNPs), namely PTPRC (rs10919563), TYK2 (rs34536443) and PRKCQ (rs4750316), which were found to be associated with JIA in previous studies. We also investigated whether the intron-4 located 27-bp VNTR of endothelial nitric oxide synthase $(e N O S)$, is associated with risk for JIA in Greece. In total, 125 JIA patients and 221 healthy controls from northern Greece were included in the study as a sample set. Samples were then analyzed, and genotyped for the three SNPs with TaqMan primer-probe sets, using a Real-Time PCR platform (ViiA ${ }^{\text {TM }} 7$ Real-Time PCR system), while eNOS VNTR polymorphism was genotyped by PCR. Statistical analysis was performed using a GraphPad Prism statistical program. The $\chi^{2}$ test was used to examine differences of genotype and allele frequencies
\end{abstract}

Correspondence to: Dr George N. Goulielmos, Section of Molecular Pathology and Human Genetics, Department of Internal Medicine, School of Medicine, University of Crete, Voutes, Heraklion 71003, Greece

E-mail: goulielmos@med.uoc.gr

${ }^{*}$ Contributed equally

Key words: juvenile idiopathic arthritis, genetic association, polymorphisms between patients and controls. Statistical significance was defined by using the two-tailed $\mathrm{P}<0.05$ test. Bioinformatics analysis was conducted by using BlastP, Pymol, Maestro and Desmond. In the case-control association study performed, eNOS only was found to be associated with JIA. Genotype $\mathrm{a} / \mathrm{a}$ and allele ' $\mathrm{a}$ ' were found in a higher frequency in JIA patients than in controls $[\mathrm{p}<0.0001$, odds ratio $(\mathrm{OR})=0.15$, 95\% confidence intervals $(\mathrm{CI}): 0.065-0.37$; and $\mathrm{p}<0.0001$, $\mathrm{OR}=0.34$, 95\% CI: 0.23-0.49, respectively]. No associations with JIA were detected for TYK2, PTPRC or PRKCQ. Aiming to investigate the structural consequences and the structure/function relationships accompanying the Pro1104 to Ala (rs34536443) mutation on TYK2 protein, bioinformatics analysis was performed. Combining three-dimensional (3D)-modeling and molecular dynamics simulations we identified changes in structural flexibility, affecting the functionality of the kinase domain of TYK2. To the best of our knowledge, this is the first time that eNOS VNTR polymorphism is associated with susceptibility to JIA, suggesting a differential role of allele 'a' in various complex diseases. The current data emphasize the importance of comparative studies in populations of a different ancestral background towards the clarification of the role of specific alleles in the development of JIA.

\section{Introduction}

Juvenile idiopathic arthritis (JIA) is a complex, autoimmune disease, characterized by persistent chronic arthritis. At present, the role of genetic and environmental factors in the development of the disease has been confirmed (1). This disorder encompasses all forms of chronic arthritis and onset appears before 16 years of age, and usually persists for more than 6 weeks (1). JIA is the most common rheumatic disease in children, with different disease manifestations in populations of different ethnicities or race. Currently, seven different subtypes of the disease have been distinguished according to 
the International League of Associations for Rheumatology (ILAR) classification (2). However, the underlying mechanisms leading to a variety of different JIA phenotypes have not been delineated yet. Nowadays, numerous suggestions have been proposed to revise this classification (3).

There is a strong genomic component to disease and a number of established susceptibility loci have been reported thus far (4-8). These data have been accumulated by using a variety of approaches, including candidate gene approaches and genome-wide association studies (GWAS). However, the pathophysiology and genetic etiology of the disease remains elusive. Most of the genetic predisposition to JIA was determined by the human leukocyte antigen (HLA) (8-10) and especially the $H L A-D R B I$ locus (10), with its contribution to be estimated at a rate of approximately $20 \%$ (11). Moreover, several non-HLA genes have been associated with JIA, including ATXN2, PTPN2, c12orf30, c3orf1, PTPN22, STAT4, TRAF1-C5, AFF3, CD247,CD226, MBL2, PSTPIP1, RANTES (CCL5), CTLA4, PRKCQ, PTPRC, TYK2, PRR9_LOR, ILDR1_CD86,WDFY4, PTH1R, RNF215, AHI1_LINC00271, JAK1, LINC00951, IL2RA, CCR5, COG6, ANGPT1, HBPI, 6q23/TNFAIP3, CXCR4 (5-7,12-19). Of these loci, TRAF1, STAT4, TNFAIP3 and PRKCQ are good candidates considering the known pathogenesis of JIA as they are located within regions known to be involved in T-cell receptor signaling or activation pathways (12). Furthermore, the role of family history regarding a higher prevalence of other autoimmune diseases among relatives of JIA patients has been confirmed (11). No genetic risk factors common for sJIA and other JIA subtypes have been reported, thus suggesting that sJIA is a unique disease process (20). A plethora of biomarkers has been suggested aiming to define the different JIA subtypes or predict either the course of the disease or treatment response (in specific therapies) (21).

Not all independent replications in various cohorts have been successful, as shown by using the ImmunoChip single nucleotide polymorphism (SNP) array (7) or by investigating an Australian cohort (15). Different genetic variations have been reported as risk factors for JIA, but the difficulty to confirm previously collected results by analyzing populations of different ethnicities indicates the existence of a heterogeneity regarding the genetic factors that are involved in JIA.

Although numerous SNPs have been associated with JIA, only a few of these studies were replicated and validated. In the present study, we aimed to validate three SNPs, namely PTPRC (rs10919563), TYK2 (rs34536443) and PRKCQ (rs4750316), previously found to be associated with JIA $(7,12,22)$ and to investigate whether the 27-bp VNTR polymorphism on intron 4 of endothelial nitric oxide synthase (eNOS), which is associated with various autoimmune diseases, is associated with risk for JIA in Greece.

\section{Patients and methods}

Patient population and study design. The sample set consisted of 125 JIA patients followed in two departments (the Rheumatology Unit of the 4th Medical Department of Internal Medicine and the Rheumatology Unit of the 1st Medical Department of Pediatrics) of the Aristotelian
University of Thessaloniki Hospital (Thessaloniki, Greece). In this study, only patients who fulfilled the 2001 revised ILAR criteria for JIA were enrolled. Written informed consent was obtained from parents for those patients who were $<18$ years of age. A total of 221 unrelated healthy individuals, sex- and ethnic-matched selected from the Transfusion Medicine Department of Hippokration Hospital of Thessaloniki, served as controls for the genotyping (14). The study was approved by the local Ethics Committee for medical research and was carried out in compliance with the Declaration of Helsinki.

Genotyping. A panel of 3 SNPs was genotyped in a Greek cohort of JIA patients and healthy controls, including PTPRC rs10919563, PRKCQ rs4750316 and TYK2 rs34536443. Moreover, the 27-bp VNTR polymorphism on intron-4 of eNOS was analyzed, considering its previous association with multiple complex diseases (23). Genomic DNA was isolated from peripheral blood leukocytes by using the commercial kit Puregene (Gentra Systems, Minneapolis, MN, USA) according to the manufacturer's instructions.

Allelic discrimination of PTPRC rs10919563, PRKCQ rs4750316 and TYK2 rs34536443 SNPs was carried out using premade TaqMan SNP Genotyping assays from Applied Biosystems (Foster City, CA, USA), according to the Applied Biosystems protocol (cat. nos. C_31565763_10,C_32036787_10 and C__60866522_10 for rs10919563, rs4750316 and rs34536443, respectively). A 96-well plate was prepared with a mixture on an Applied Biosystems ViiA ${ }^{\mathrm{TM}} 7$ Real-Time PCR system of 1X TaqMan SNP Genotyping assay, 1X TaqMan Universal Master mix (both from Applied Biosystems) and 20 ng DNA per well. After PCR, the plates were read, and the data analyzed using Quant Studio ${ }^{\text {TM }}$ Real-Time PCR software (Applied Biosystems). Each assay was run with negative controls. For quality control, a random $10 \%$ of the samples were amplified twice to ensure accuracy of the results, with a reproducibility rate of $100 \%$. Genotyping of $e N O S$ gene intron $4 \mathrm{a} / \mathrm{b}$ VNTR polymorphism was performed as described in detail elsewhere (23). Upon PCR amplification, the 420-bp band indicated five repeats of the 27-bp sequence and the 393-bp band represented four repeats, corresponding to $b$ and a alleles, respectively (24).

Construction of a three-dimensional (3D) model. A bioinformatics analysis was performed using BlastP (for sequence analysis) on the Uniprot sequence database. PyMOL (DeLano Scientific, San Carlos, CA, USA) was used for the direction of the development of a 3D model. Maestro and Desmond (Schrödinger, Inc., New York, NY, USA) were used aiming to analyze mutations and stability of the protein model. The initial 3D model used was based on the crystal structure of Homo sapiens TYK2.

Statistical analysis. The GraphPad Prism statistical program (GraphPad Software, San Diego, CA, USA) was used in the framework of the analyses conducted. A two-tailed $\mathrm{P}<0.05$ was defined as statistically significant. Odds ratios (OR) and 95\% confidence intervals (CI) were calculated. The possible deviation from Hardy-Weinberg equilibrium (HWE) was performed by using the program 'Calculate' (Copyright TRG, SR, INMD, 2008). 
Table I. Genotype and allele frequencies of the 27-bp eNOS VNTR analyzed in 125 JIA patients and 221 healthy controls.

\begin{tabular}{lcccc}
\hline Variables & JIA $(\mathrm{N}=125)$ & Controls $(\mathrm{N}=221)$ & P-value & OR (95\% CI) \\
\hline Genotypes & & & & \\
b/b & $60(48 \%)$ & $162(73.30 \%)$ & & 0.05 \\
b/a & $47(37.6 \%)$ & $49(22.17 \%)$ & $\mathbf{< 0 . 0 0 0 1}$ & $0.15(0.16-1.065-0.37)$ \\
a/a & $19(15.2 \%)$ & $8(3.63 \%)$ & & \\
Alleles & & & & \\
B & $167(66.8 \%)$ & $373(84.39 \%)$ & $\mathbf{< 0 . 0 0 0 1}$ & $0.34(0.23-0.49)$ \\
A & $85(33.2 \%)$ & $65(14.71 \%)$ & \\
\hline
\end{tabular}

eNOS, endothelial nitric oxide synthase; JIA, juvenile idiopathic arthritis; OR, odds ratio; CI, confidence interval. Bold, statistically significant.

\section{Results}

Developing a $3 D$ model of TYK2 protein. Aiming to gain insight to the potential structural/functional consequences of rs34536443 SNP, which has been associated with various rheumatic and other complex human diseases, we further analyzed the respective region of TYK2 protein by developing a new 3D model, improving the pre-existing one developed by Myrthianou et al (26). The rs34536443 SNP leads to the substitution of a $\mathrm{G}$ to a $\mathrm{C}$ nucleotide, thus resulting in a Pro1104Ala mutation in the TYK2 protein. Accordingly, replacement of the Pro1104 amino acid residue of the protein TYK2 may introduce a conformational change that may extend the $\alpha$-helical segment by five more residues (Fig. 1). Thus, it is possible that the local 3D structure may be altered and, consequently, the folding of TYK2 protein may be affected. These alterations are supposed to affect the protein from the functionality viewpoint. Subsequent analyses of molecular dynamics of the mutant TYK2 demonstrated further folding changes at the level of the structure of the protein (Fig. 1).

Investigating a potential genetic association of TYK2, PTPRC and eNOS SNPS with JIA. In the context of this case-control study, three successful markers were analyzed. The allelic and genotype frequencies of the eNOS VNTR of patients and controls are shown in Table I. The homozygous genotype $\mathrm{b} / \mathrm{b}$ appeared the higher frequency in controls as well as JIA patients ( 73.30 vs. $48 \%$, respectively). Importantly, a/a genotypes had a frequency that was associated with disease occurrence $(\mathrm{p}<0.0001, \mathrm{OR}=0.15,95 \%$ CI: $0.065-0.37)$. Moreover, the frequency of the eNOS intron-4 'a' allele of JIA patients was associated with the disease, being higher in comparison to that of the controls (33.2 vs. $14.71 \%$; $p<0.0001$, $\mathrm{OR}=0.34,95 \%$ CI: $0.23-0.49)$. These data indicated the 27-bp eNOS VNTR with JIA in Greece.

Notably, the remaining two SNPs PTPRC (rs10919563) and TYK2 (rs34536443) were found to be associated with JIA apart from previous findings that suggested a genetic association between these SNPs and JIA in different ethnic populations $(6,22)$. Thus, patients with JIA did not present higher frequencies either for the GG genotype or $\mathrm{G}$ allele of the PTPRC rs10919563 SNP ( $\mathrm{p}=0.41, \mathrm{OR}=0.3$,

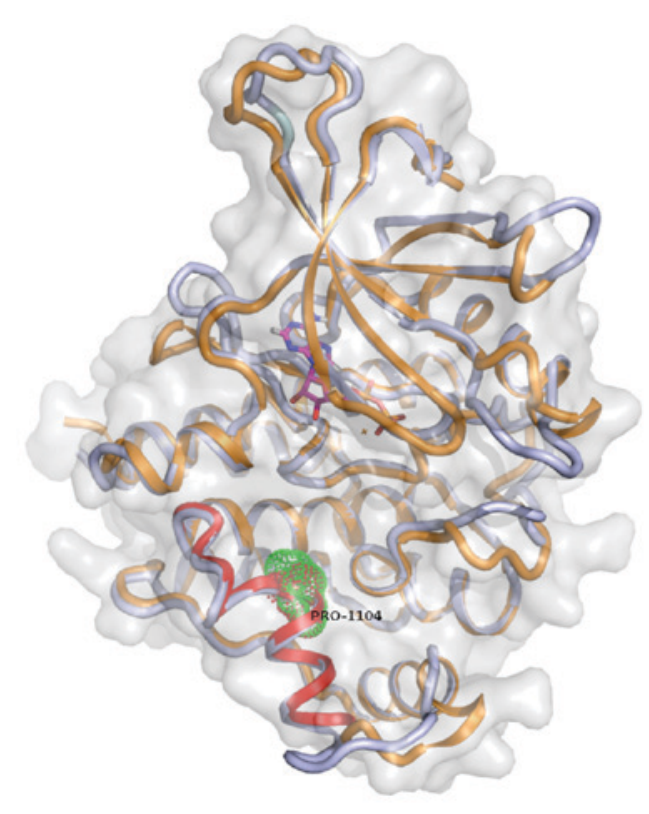

Figure 1. 3D representation both in volume and backbone of the TYK2 native structure (PDB code $4 \mathrm{GVJ}$ ) (in brown) and the mutant model (in blue-grey) containing the position of the P1104A polymorphism (green). Helical segment $1099-1113$ is in red. 3D, three dimensional.

95\% CI: 0.03-2.6; and $\mathrm{p}=0.49$, OR=0.82, 95\% CI: 0.52-1.31, respectively) (data not shown). Moreover, concerning the TYK2 rs34536443 SNP, no statistical significant differences were observed between patients and controls either at genotype or allelic level $(\mathrm{p}=0.34$, OR=2.50, 95\% CI: $1.53-11.75$; and $\mathrm{p}=0.34$, OR $=2.45,95 \% \mathrm{CI}: 0.53-11.55$ for $\mathrm{G} / \mathrm{C}$ genotype and minor allele $\mathrm{C}$, respectively) (data not shown). All the gene polymorphisms examined were found to be under HWE $(\mathrm{P}>0.01)$.

In our attempt to analyze a possible association of the polyarticular and oligoarticular course of JIA with the two SNPs and the 27-bp VNTR under study, a further genetic analysis was conducted but no association was observed. Considering the moderate size of our sample, which precluded any detailed subtype-specific association analyses, it is understood that such a subtype analysis must be conducted in a larger cohort, consisted of adequately sized samples, in order to lead to a high statistical power. 
Table II. Frequencies of the minor allele 'C' of $P R K C Q$ rs4750316 SNP in a sex-stratified genetic analysis.

\begin{tabular}{lllccr}
\hline Gene & SNP & Samples & N cases/N controls & OR (95\% CI) & P-value \\
\hline PRKCQ & \multirow{2}{*}{ rs4750316 } & All & $144 / 185$ & $1.12(0.75-1.68)$ & 0.62 \\
& & Males & $36 / 129$ & $1.80(0.88-3.64)$ & 0.14 \\
& & Females & $108 / 56$ & $0.61(0.32-1.17)$ & 0.16 \\
\hline
\end{tabular}

Search for a sex-specific genetic association of PRKCQ rs4750316 SNP with JIA. A genetic association study was performed involving the $P R K C Q$ rs4750316 SNP (Table II). However, no significant association of the minor allele $\mathrm{C}$ of rs4750316 with JIA was observed $(\mathrm{p}=0.62$, OR=1.12, 95\% CI: 0.75-1.68). Nevertheless, preliminary unpublished data collected from different ethnic populations have previously shown $P R K C Q$ rs 4750316 SNP to be associated with JIA specifically to males. Therefore, a sex-stratified analysis was conducted in the Greek samples but no sex-based difference in the frequency of this allele was observed between patients ( $\mathrm{p}=0.14, \mathrm{OR}=1.80,95 \%$ CI: $0.88-3.64$ vs. $\mathrm{p}=0.16, \mathrm{OR}=0.61$, 95\% CI: 0.32-1.17, respectively). Given that the sex bias differs in the JIA subtype, these associations should be further investigated in individual subtypes. However, the small sample size did not allow for further analyses, depending on JIA subtype at level of a statistical certainty, to be performed.

\section{Discussion}

Accumulated evidence has indicated the existence of a heterogeneity, depending on the population's ethnicity, of genetic factors involved in the development of autoimmune diseases. Deepening of our knowledge regarding the homogeneity or heterogeneity at the level of alleles that is associated with these diseases may confer to a more reasonable understanding of plausible common gene function and pathways. To the best of our knowledge, the present analysis revealed for the first time that eNOS VNTR polymorphism is associated with susceptibility to JIA and, therefore, suggests a role for the risk allele ' $a$ ' in the development of different autoimmune diseases. In a previous study, referring to a Greek population, the implication of $e N O S$ gene 27-bp VNTR in susceptibility to rheumatoid arthritis (RA) was identified (23). Moreover, in that study, the implication of this VNTR to systemic lupus erythematosus (SLE) was shown (23). In the present study, it was found that the presence of $a / b$ genotype or 'a' allele may be a disease-susceptibility factor for RA or lupus glomerulonephritis.

The functional role of nitric oxide (NO) regarding the cell pathophysiology and function has been previously analyzed. Thus, it has been suggested that it is a potent mediator in different biological reactions, which is involved in various responses based either on inflammation or autoimmunity processes. NO was suggested to be an inhibiting factor for proinflammatory gene expression in endothelial cells or an activator of resident leukocytes (27). Obviously, gene polymorphisms or other factors that influence the expression of the enzyme eNOS that catalyzes the synthesis of NO can be considered as key genes for the control of NO levels activity. For the maintenance of physiological endothelial function, low levels of NO are needed (28). Although the functional significance of $e N O S$ gene VNTR on protein activity and expression is not well documented yet, some preliminary experiments have managed to clarify this issue. The a allele has been related to low NO metabolite levels, and a/a homozygotes were found to produce lower NO metabolite levels compared to $b / b$ individuals (29). In addition, it has been shown that the 27-bp VNTR of $e N O S$ is able to bind nuclear proteins acting either as an enhancer or repressor, thus promoting or suppressing transcription efficiency (30). Therefore, apart from the suggested crucial role of the $e N O S$ VNTR under discussion dealing with the decreased gene expression of $e N O S$ in allele 'a' carriers, the possibility of its proximity and, therefore, of a linkage disequilibrium with other variants cannot be underestimated.

An association of $e N O S$ gene with various multifactorial diseases, including as primary biliary cirrhosis (31), RA (23), type- 1 and -2 diabetes $(32)$ and $\operatorname{SLE}(23,33)$ has been shown thus far. However, a dependence of JIA and eNOS polymorphisms according to race or ethnicity has been suggested. Indeed, an association of the eNOS VNTR polymorphism with SLE was shown in the past in Turkey (33) but not in Greece (23) or with RA in Greece (23) but not in Serbia (34).

Notably, although it was previously shown that PTPRC rs10919563 (6), PRKCQ rs4750316 (12) and TYK2 rs34536443 (22) SNPs play a role in the phenotypical manifestations accompanying JIA, the present study did not confirm association of the SNPs examined with the disease in Greece.

It is well known that the high female:male sex-ratio is a characteristic observed in almost all autoimmune diseases, including JIA. Recently, a sex-specific pattern of association with JIA has been reported. Particularly, a female-association of PTPN22 rs2476601 was detected with JIA in different ethnic populations, including the Greek one (35). In this framework, the rs4750316 SNP of $P R K C Q$ was analyzed as well, taking into account that it was demonstrated originally to exhibit an association with JIA in a first analysis conducted in 2010 (12), thus appearing as a promising JIA candidate gene in the UK cohort. However, this finding was not confirmed in the same population when an analysis by ImmunoChip array was performed (7). Notably, an association of this SNP with JIA was found recently in samples from different ethnicities, stratified by sex (unpublished data). While in the Greek population an analysis for rs4750316 SNP did not reach statistical significance, more solid data are to be obtained by increasing the sample size and further investigating any new data.

It is worth mentioning that a study performed by using the Immunochip and next generation sequencing approaches has shown that allele ' $\mathrm{C}$ ' of rs 34536443 of $T Y K 2$ was protective for inflammatory bowel disease, RA and SLE (36) but conferred a risk for psoriasis (37) and JIA (6). In the framework of an 
analysis conducted in a UK cohort by ImmunoChip array (7), TYK2 rs34536443 was not found to be associated with JIA, a result that coincides with the present data from the Greek cohort. This finding was noteworthy considering that $T Y K 2$ rs34536443 SNP was found recently, in a study performed in the Greek population (26), to exhibit a genetic overlap with PsA and RA that is well known to possess or share several similar clinical manifestations with JIA. Moreover, according to the constructed 3D model of TYK2 protein and previous observations of our group (26), it is assumed that the Pro1104Gly mutation may affect the protein's functionality by affecting elements contributing to the structural conformation of the molecule.

According to the data of the present study, minor allele frequencies (MAFs) of PRKCQ (rs4750316), TYK2 (rs34536443) SNPs and $e N O S$ VNTR were lower in healthy individuals from Greece in comparison to samples analyzed by other investigators working with different ethnic/racial populations $(6,14,22,37,38)$. This finding suggests that existing population-specific differences influence the frequency of these alleles. Thus, there is still the possibility that an association of TYK2 rs34536443 with JIA may be detected if the cohort size is increased. However, given the MAF of the minor allele C of this SNP in the controls from Greece (0.22), it can be assumed that a high number of patients and controls was required to be collected (probably higher than 5,000 for each category) to obtain an $80 \%$ power of the study (at $\mathrm{p}=0.05$ ) (26).

Findings of this study highlight the importance of conducting comparative studies in different populations, considering that replication of previously identified markers is paramount to determine which SNPs are true risk loci, thus suggesting key disease pathways which require further study. Additionally, the ultimate task upon a proper functional validation of these SNPs is the translation of any findings into a better management of specific groups of patients with JIA.

\section{Acknowledgements}

We would like to thank Dr J. Ellis and Dr R.C. Chiaroni-Clarke (Victoria, Australia) for the constructive discussions concerning the role of $P R K C Q$ gene polymorphism in JIA.

\section{References}

1. Ravelli A and Martini A: Juvenile idiopathic arthritis. Lancet 369: 767-778, 2007.

2. Petty RE, Southwood TR, Manners P, Baum J, Glass DN, Goldenberg J, He X, Maldonado-Cocco J, Orozco-Alcala J, Prieur AM, et al; International League of Associations for Rheumatology: International League of Associations for Rheumatology classification of juvenile idiopathic arthritis: Second revision, Edmonton, 2001. J Rheumatol 31: 390-392, 2004.

3. Martini A: It is time to rethink juvenile idiopathic arthritis classification and nomenclature. Ann Rheum Dis 71: 1437-1439, 2012.

4. Thompson SD, Sudman M, Ramos PS, Marion MC, Ryan M, Tsoras M, Weiler T, Wagner M, Keddache M, Haas JP, et al: The susceptibility loci juvenile idiopathic arthritis shares with other autoimmune diseases extend to PTPN2, COG6, and ANGPT1. Arthritis Rheum 62: 3265-3276, 2010.

5. Thompson SD, Marion MC, Sudman M, Ryan M, Tsoras M, Howard TD, Barnes MG, Ramos PS, Thomson W, Hinks A, et al: Genome-wide association analysis of juvenile idiopathic arthritis identifies a new susceptibility locus at chromosomal region 3q13. Arthritis Rheum 64: 2781-2791, 2012.
6. Hinks A, Cobb J, Sudman M, Eyre S, Martin P, Flynn E, Packham J, Barton A, Worthington J, Langefeld CD, et al; Childhood Arthritis Prospective Study (CAPS); UK RA Genetics (UKRAG) Consortium; British Society of Paediatric and Adolescent Rheumatology (BSPAR) Study Group: Investigation of rheumatoid arthritis susceptibility loci in juvenile idiopathic arthritis confirms high degree of overlap. Ann Rheum Dis 71: 1117-1121, 2012.

7. Hinks A, Cobb J, Marion MC, Prahalad S, Sudman M, Bowes J, Martin P, Comeau ME, Sajuthi S, Andrews R, et al; Boston Children's JIA Registry; British Society of Paediatric and Adolescent Rheumatology (BSPAR) Study Group; Childhood Arthritis Prospective Study (CAPS); Childhood Arthritis Response to Medication Study (CHARMS); German Society for Pediatric Rheumatology (GKJR); JIA Gene Expression Study; NIAMS JIA Genetic Registry; TREAT Study; United Kingdom Juvenile Idiopathic Arthritis Genetics Consortium (UKJIAGC): Dense genotyping of immune-related disease regions identifies 14 new susceptibility loci for juvenile idiopathic arthritis. Nat Genet 45: 664-669, 2013.

8. Hinks A, Bowes J, Cobb J, Ainsworth HC, Marion MC, Comeau ME, Sudman M, Han B, Becker ML, Bohnsack JF, et al; Juvenile Arthritis Consortium for Immunochip: Fine-mapping the MHC locus in juvenile idiopathic arthritis (JIA) reveals genetic heterogeneity corresponding to distinct adult inflammatory arthritic diseases. Ann Rheum Dis 76: 765-772, 2017.

9. Thomson W, Barrett JH, Donn R, Pepper L, Kennedy LJ, Ollier WE, Silman AJ, Woo P and Southwood T; British Paediatric Rheumatology Study Group: Juvenile idiopathic arthritis classified by the ILAR criteria: HLA associations in UK patients. Rheumatology (Oxford) 41: 1183-1189, 2002.

10. Hollenbach JA, Thompson SD, Bugawan TL, Ryan M, Sudman M, Marion M, Langefeld CD, Thomson G, Erlich HA and Glass DN: Juvenile idiopathic arthritis and HLA class I and class II interactions and age-at-onset effects. Arthritis Rheum 62: 1781-1791, 2010.

11. Prahalad S, Shear ES, Thompson SD, Giannini EH and Glass DN: Increased prevalence of familial autoimmunity in simplex and multiplex families with juvenile rheumatoid arthritis. Arthritis Rheum 46: 1851-1856, 2002.

12. Hinks A, Eyre S, Ke X, Barton A, Martin P, Flynn E, Packham J, Worthington J and Thomson W; Childhood Arthritis Prospective Study (CAPS); UKRAG Consortium; BSPAR Study Group: Overlap of disease susceptibility loci for rheumatoid arthritis and juvenile idiopathic arthritis. Ann Rheum Dis 69: 1049-1053, 2010.

13. Hinks A, Eyre S, Ke X, Barton A, Martin P, Flynn E, Packham J, Worthington J and Thomson W; Childhood Arthritis Prospective Study; UKRAG Consortium; BSPAR Study Group: Association of the AFF3 gene and IL2/IL21 gene region with juvenile idiopathic arthritis. Genes Immun 11: 194-198, 2010b.

14. Dimopoulou DG, Zervou MI, Trachana M, Myrthianou E, Pratsidou-Gertsi P, Kardassis D, Garyfallos A and Goulielmos GN: Investigation of juvenile idiopathic arthritis susceptibility loci: Results from a Greek population. Hum Immunol 74: 1194-1198, 2013.

15. Ellis JA, Chavez RA, Pezic A, Ponsonby AL, Akikusa JD, Allen RC and Munro JE: Independent replication analysis of genetic loci with previous evidence of association with juvenile idiopathic arthritis. Pediatr Rheumatol Online J 11: $12,2013$.

16. Herlin MP, Petersen MB and Herlin T: Update on genetic susceptibility and pathogenesis in juvenile idiopathic arthritis. EMJ Rheumatol 1: 73-83, 2014.

17. Singh S, Bhattad S and Danda D: Genetics of juvenile idiopathic arthritis. Int J Rheum Dis 17: 233-236, 2014.

18. Finkel TH, Li J, Wei Z, Wang W, Zhang H, Behrens EM, Reuschel EL, Limou S, Wise C, Punaro M, et al: Variants in CXCR4 associate with juvenile idiopathic arthritis susceptibility. BMC Med Genet 17: 24, 2016.

19. McIntosh LA, Marion MC, Sudman M, Comeau ME, Becker ML, Bohnsack JF, Fingerlin TE, Griffin TA, Haas JP, Lovell DJ, et al; Boston Children's JIA Registry; German Society for Pediatric Rheumatology (GKJR); JIA Gene Expression Studies; NIAMS JIA Genetic Registry; TREAT Study; Understanding TNF Therapy in JIA Project: Genome-wide association meta-analysis reveals novel juvenile idiopathic arthritis susceptibility loci. Arthritis Rheumatol: July 18, 2017 (Epub ahead of print). 
20. Ombrello MJ, Arthur VL, Remmers EF, Hinks A, Tachmazidou I, Grom AA, Foell D, Martini A, Gattorno M, Özen S, et al; British Society of Pediatric and Adolescent Rheumatology (BSPAR) Study Group, Inception Cohort of Newly Diagnosed Patients with Juvenile Idiopathic Arthritis (ICON-JIA) Study Group, Childhood Arthritis Prospective Study (CAPS) Group, Randomized Placebo Phase Study of Rilonacept in sJIA (RAPPORT) Investigators, Sparks-Childhood Arthritis Response to Medication Study (CHARMS) Group, Biologically Based Outcome Predictors in JIA (BBOP) Group: Genetic architecture distinguishes systemic juvenile idiopathic arthritis from other forms of juvenile idiopathic arthritis: Clinical and therapeutic implications. Ann Rheum Dis 76: 906-913, 2017.

21. Consolaro A, Varnier GC, Martini A and Ravelli A: Advances in biomarkers for paediatric rheumatic diseases. Nat Rev Rheumatol 11: 265-275, 2015.

22. Tao JH, Zou YF, Feng XL, Li J, Wang F, Pan FM and Ye DQ: Meta-analysis of TYK 2 gene polymorphisms association with susceptibility to autoimmune and inflammatory diseases. Mol Biol Rep 38: 4663-4672, 2011.

23. Vazgiourakis V1, Sidiropoulos P, Bertsias G, Koutsounaki E, Fragouli E, Raptopoulou A, Kritikos H, Boumpas DT and Goulielmos GN: Association of the nitric oxide synthase (eNOS) gene polymorphism with increased risk for both lupus glomerulonephritis and rheumatoid arthritis in a single genetically homogeneous population. Lupus 16: 867-874, 2007.

24. Miyahara K, Kawamoto T, Sase K, Yui Y, Toda K, Yang LX, Hattori R, Aoyama T, Yamamoto Y, Doi Y, et al: Cloning and structural characterization of the human endothelial nitric-oxidesynthase gene. Eur J Biochem 223: 719-726, 1994.

25. Liang J, Tsui V, Van Abbema A, Bao L, Barrett K, Beresini M, Berezhkovskiy L, Blair WS, Chang C, Driscoll J, et al: Lead identification of novel and selective TYK2 inhibitors. Eur J Med Chem 67: 175-187, 2013.

26. Myrthianou E, Zervou MI, Budu-Aggrey A, Eliopoulos E, Kardassis D, Boumpas DT, Kougkas N, Barton A, Sidiropoulos P and Goulielmos GN: Investigation of the genetic overlap between rheumatoid arthritis and psoriatic arthritis in a Greek population. Scand J Rheumatol 46: 180-186, 2017.

27. Guzik TJ, Korbut R and Adamek-Guzik T: Nitric oxide and superoxide in inflammation and immune regulation. J Physiol Pharmacol 54: 469-487, 2003.

28. Albrecht ED, Aberdeen GW, Niklaus AL, Babischkin JS, Suresch DL and Pepe GJ: Acute temporal regulation of vascular endothelial growth/permeability factor expression and endothelial morphology in the baboon endometrium by ovarian steroids. J Clin Endocrinol Metab 88: 2844-2852, 2003.

29. Noiri E, Satoh H, Taguchi J, Brodsky SV, Nakao A, Ogawa Y, Nishijima S, Yokomizo T, Tokunaga K and Fujita T: Association of eNOS Glu298Asp polymorphism with end-stage renal disease. Hypertension 40: 535-540, 2002.
30. Wang J, Dudley D and Wang XL: Haplotype-specific effects on endothelial NO synthase promoter efficiency: Modifiable by cigarette smoking. Arterioscler Thromb Vasc Biol 22: e1-e4, 2002.

31. Mantaka A, Goulielmos GN, Koulentaki M, Tsagournis O, Voumvouraki A and Kouroumalis EA: Polymorphisms of genes related to endothelial cells are associated with primary biliary cirrhosis patients of Cretan origin. Hum Immunol 73: 829-835, 2012.

32. Galanakis E1, Kofteridis D, Stratigi K, Petraki E, Vazgiourakis V, Fragouli E, Mamoulakis D, Boumpas DT and Goulielmos GN: Intron $4 \mathrm{a} / \mathrm{b}$ polymorphism of the endothelial nitric oxide synthase gene is associated with both type 1 and type 2 diabetes in a genetically homogeneous population. Hum Immunol 69: 279-283, 2008.

33. Zervou MI, Vazgiourakis VM, Yilmaz N, Kontaki E, Trouw LA, Toes RE, Bicakcigil M, Boumpas DT, Yavuz S and Goulielmos GN: TRAF1/C5, eNOS, C1q, but not STAT4 and PTPN22 gene polymorphisms are associated with genetic susceptibility to systemic lupus erythematosus in Turkey. Hum Immunol 72: 1210-1213, 2011.

34. Bunjevacki V, Maksimovic N, Jekic B, Milic V, Lukovic L, Novakovic I, Damjanov N, Radunovic G and Damnjanovic T: Polymorphisms of the eNOS gene are associated with disease activity in rheumatoid arthritis. Rheumatol Int 36: 597-602, 2016.

35. Goulielmos GN, Chiaroni-Clarke RC, Dimopoulou DG, Zervou MI, Trachana M, Pratsidou-Gertsi P, Garyfallos A and Ellis JA: Association of juvenile idiopathic arthritis with PTPN22 rs2476601 is specific to females in a Greek population. Pediatr Rheumatol Online J 14: 25, 2016.

36. Diogo D, Bastarache L, Liao KP, Graham RR, Fulton RS, Greenberg JD, Eyre S, Bowes J, Cui J, Lee A, et al: TYK2 protein-coding variants protect against rheumatoid arthritis and autoimmunity, with no evidence of major pleiotropic effects on non-autoimmune complex traits. PLoS One 10: $\mathrm{e} 0122271,2015$.

37. Tsoi LC, Spain SL, Knight J, Ellinghaus E, Stuart PE, Capon F, Ding J, Li Y, Tejasvi T, Gudjonsson JE, et al; Collaborative Association Study of Psoriasis (CASP); Genetic Analysis of Psoriasis Consortium; Psoriasis Association Genetics Extension; Wellcome Trust Case Control Consortium 2: Identification of 15 new psoriasis susceptibility loci highlights the role of innate immunity. Nat Genet 44: 1341-1348, 2012.

38. Neugebauer S, Baba T and Watanabe T: Association of the nitric oxide synthase gene polymorphism with an increased risk for progression to diabetic nephropathy in type 2 diabetes. Diabetes 49: 500-503, 2000 . 